\title{
The Evolution of the \\ World Health \\ Organization (WHO) \\ Manual on Semen
}

Analysis

Janet Nzisa

Editorial Assistant

Citation: EMJ Repro Health. 2021;7[1]:23-26.
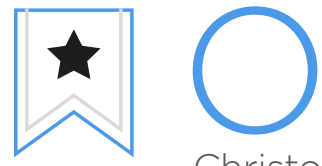
N DAY 4 of the European Society of Human Reproduction and Embryology (ESHRE) Congress 2021, participants worldwide virtually joined a session on semen analysis (SA) entitled "Evolution of the WHO Manual: Where Are We Now?" chaired by Christopher De Jonge, Director of the Andrology Programme at the University of Minnesota Medical Center, USA, and Ellen Goossens, Professor at Vrije Universiteit Brussel, Belgium.

\section{EVOLUTION OF THE WHO MANUAL}

Sandro Esteves, Medical Director at ANDROFERT Clinic, Campinas, Brazil, began the session by defining male infertility as the disease that affects the male reproductive system that is primarily caused by genetic and congenital conditions, anatomical, endocrine, functional or immunological abnormalities, chronic illness, certain lifestyle factors, and sexual-related conditions. These factors could be associated with semen deficiencies and may not be detected during SA. Currently, routine SA is the only test carried out to determine an individual's fertility potential. However, male fertility evaluation solely based on the results of routine SA is inadequate in the identification of underlying conditions associated with infertility. A recently published study by Esteves explicated that male fertility primarily detected through routine SA does not provide males with an optimal fertility pathway. Firstly, the reference intervals in routine SA do not accurately distinguish fertility from subfertility. Secondly, the results procured from an individual, unless at extremely lower limits, have a restricted prognostic value in both natural and assisted conception. Furthermore, routine SA does not detect the defects in sperm DNA that may adversely influence embryo implantation, development, and overall offspring health.

The European Association of Urology (EAU) 2021 guidelines state that male and female partners should be simultaneously assessed during the initial fertility evaluation. The evaluation in males should not be limited to SA only but should also include a medical and reproductive history, 
physical examination, sperm function test, and endocrine evaluation. Additional testing such as genetic evaluation, transrectal ultrasound, retrograde ejaculation assessment, testicular biopsy, and vasography may be required depending on the clinical characteristics and semen parameters of the individual. A study by Schlegel et al. states that the objectives of a male fertility work-up are to identify the following types of conditions:

\section{> a potentially correctable condition;}

$>$ irreversible conditions suitable for assisted reproductive technology (ART) using the individual's sperm;

$>$ conditions in which donor insemination or adoptions are the main options;

$>$ any genetic abnormalities that may affect the health of a patient or offspring, especially if ART is used.

Esteves emphasised that SA is fundamental in the evaluation of the male fertility component and provides valuable information about spermatogenesis, epididymides, and the accessory sexual glands. Furthermore, SA could help in the diagnosis of defects in the male reproductive organs, follow-up in male infertility treatment, and even the selection of suitable ART treatment modality. SA is a highly complex laboratory test and therefore should be carried out exclusively in an andrology laboratory by experienced technicians. Additionally, internal and external quality controls, validation of test systems, and quality assurance are aspects to be considered when choosing a laboratory to carry out SA.

Since the 1980s, the Department of Reproductive Health and Research of the World Health Organization (WHO) has been publishing guidelines on how the assessment of human semen should be carried out in the laboratory. These guidelines are updated periodically and incorporate the latest evidence derived from research. The manual for the examination and processing of human semen includes a detailed protocol, reference standard values, and other relevant information. The current fifth edition of the manual was published in 2010 and can be found on the WHO website. This manual contains guidelines for the examination and processing of human semen utilised by technicians in clinical and research laboratories and consists of three main sections: SA, sperm preparation, and quality assurance.

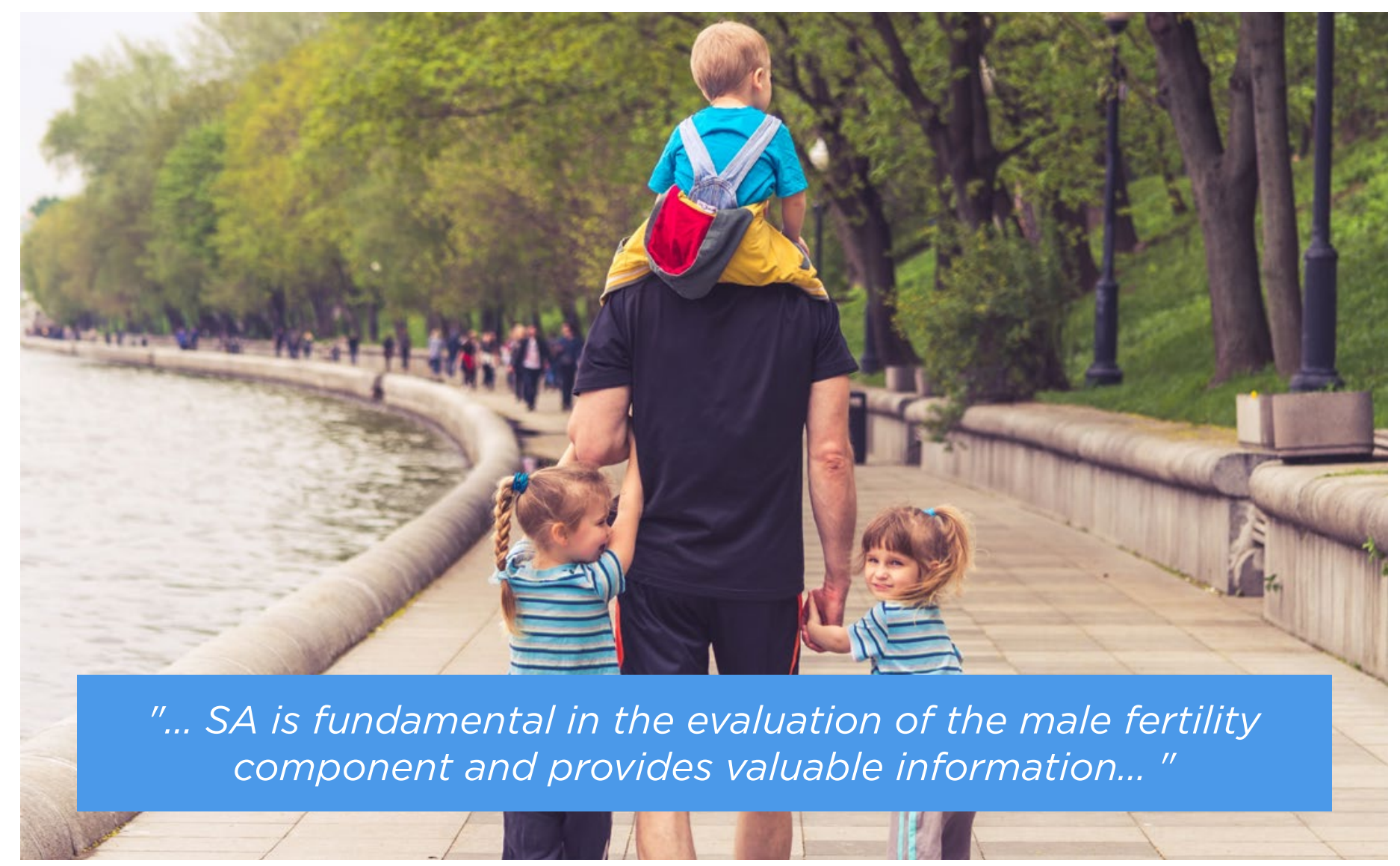


Esteves explained that the authors of the fifth edition WHO guidelines on human sperm assessments relied on clinical chemistry principles to generate $95 \%$ intervals for sperm volume, count, motility, vitality, and morphology from recent fathers ( $\leq 12$ months). A total of 1,953 individual male sperm specimens were used to generate these data. These values have been used in clinical practice and research; however, when compared to previous editions of the WHO manuals, the 2010 standards were marked lower. The previous editions relied on the clinical experience of the authors who had studied populations of males considered healthy and fertile without the presence of a pregnancy. Therefore, the quality of data used in previous WHO editions to generate reference values could not be contended and therefore was not reliable. Nevertheless, the reference values of the fifth edition have raised concerns about its clinical utility and general practicality and have been the subject of numerous debates. Criticisms include the limited geographical area of the participants, the methods used in SA, and the potential impact of the new reference ranges. Esteves further explained that the data used to generate the 2010 WHO manual references values were most exclusively collected from participants who lived in countries located in the northern hemisphere. Furthermore, the data used to produce these values were from a group of investigators who worked collaboratively, therefore it is not clear to what extent the information represents the global semen characteristics of a male who is considered to be fertile. Nearly $40 \%$ of the male infertility population previously classified as having abnormal levels of SA values based on the 1999 WHO manual reference values were reclassified as having SA references values within the normal range in the 2010 WHO manual guidelines. These results further highlight that male infertility testing primarily based on routine SA does not provide a guide towards an optimal fertility pathway.

\section{SPERM DNA FRAGMENTATION TESTING}

Esteves presented a meta-analysis study that his team carried out on the effect of cigarette smoking on semen quality, to analyse the impact of 2010 WHO guidelines of SA. The results showed that smoking negatively impacted sperm morphology when the reference values of the 1999 or earlier WHO manuals were used; however, this was not the case when the 2010 WHO manual methodology and reference values were used. As stated by Esteves, approximately $30 \%$ of males with unexplained male infertility present with sperm deficiencies that can only be recognised through sperm functionality examinations such as assessment of DNA integrity and oxidative stress tests. In another study by Esteves et al., 70\% of patients had high sperm DNA fragmentations despite having sperm parameters within the normal range according to the WHO manual guidelines. Therefore, it is clear that integration of other tests alongside the routine SA tests are required and may add valuable information in fertility counselling, diagnosis, and treatment planning. The sperm DNA fragmentation tests could assist in identifying underlying conditions that affect male fertility. Another study by Esteves showed that sperm DNA fragmentation could be treated by avoiding factors that contribute to oxidative stress such as smoking, obesity, and environmental factors.

Oral antioxidant therapies and supplements such as vitamin $C$ and E, folic acid, selenium, zinc, and co-enzyme Q10, among others, could be beneficial in improving male fertility. Another approach involves the treatment of underlying conditions that cause male infertility, e.g., genital tract infections, leukocytospermia, varicocele repair, diabetes, thyroid disorders, and hyperprolactinaemia. Follicle stimulating hormone therapy is advantageous in the regulation and production of sperm in males affected by infertility. Furthermore, intracytoplasmic sperm injection and ART are effective interventions for men affected by infertility. Esteves emphasised that fertility specialists, reproductive urologists, and clinical andrologists should perform male fertility evaluations by carrying out a detailed history examination, physical examination, routine SA, and other tests as needed.

\section{WHO MANUAL UPDATE 2021}

The upcoming sixth edition of the $\mathrm{WHO}$ laboratory manual for the examination and processing of human semen includes essential updates. Esteves stated the draft was made available in March 2021 for public review, and he compared it to the previous editions. Chapters 


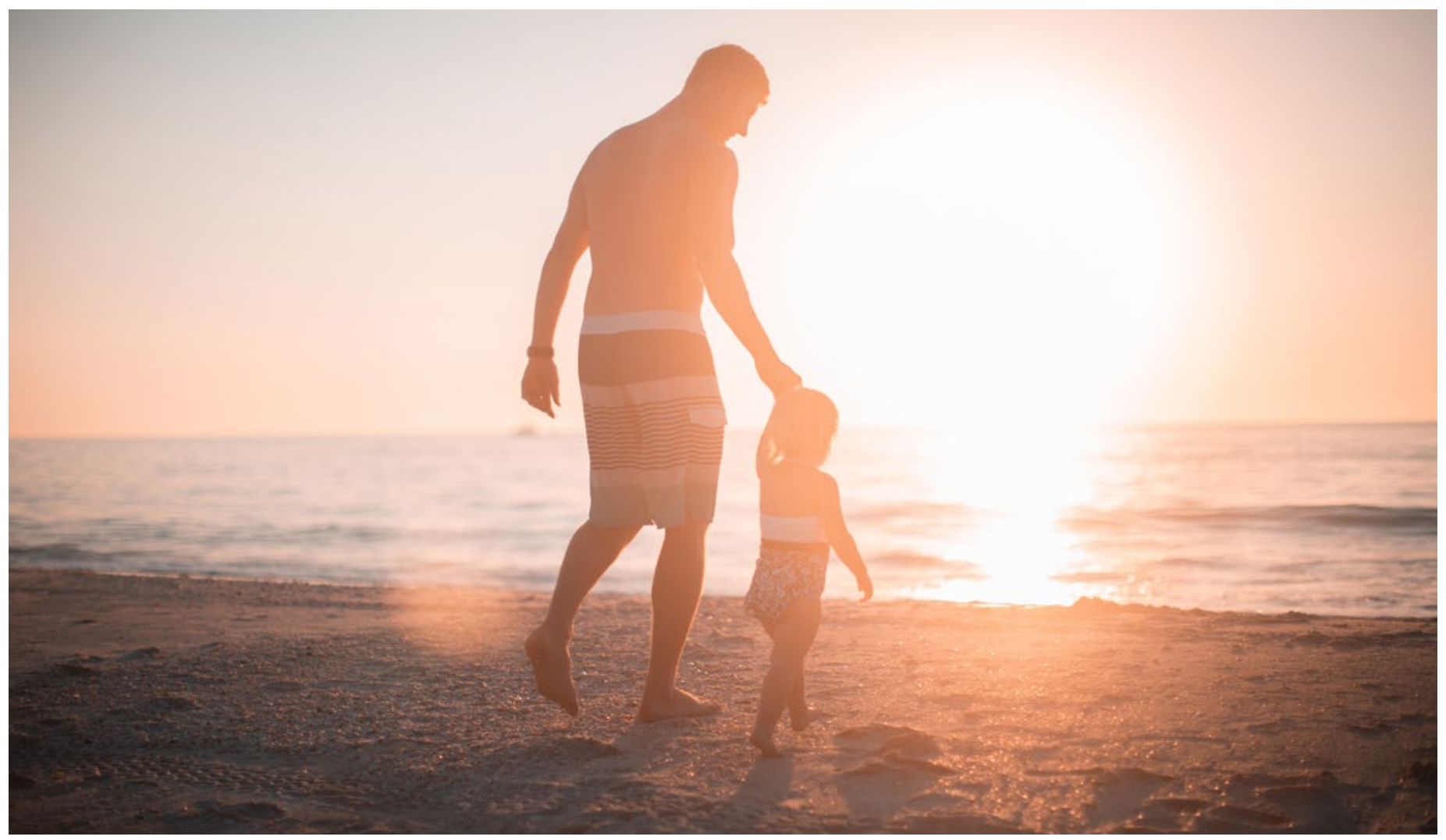

on tests that are no longer essential like sperm cervical penetration, assays, and hamster egg penetration have been removed. Alternatively, the advanced tests section has been extensively improved with comprehensive information covering sperm DNA fragmentation tests, markers for genital tract inflammation, sequence for ejaculation, semen biochemistry, and sperm aneuploidy assessments, among other updates. Research procedures such as oxidative stress test, acrosome reaction test, and sperm chromatin structure and stability procedures have also been extensively covered. Furthermore, Esteves highlighted that the sections related to sperm processing, cryopreservation of spermatozoa, and quality control and assurance are now more detailed. Each of these updated sections contains a variety of protocols for processing both ejaculated and surgically extracted sperm and includes details on sperm vitrification techniques.

Additionally, novel SA data have been included in the draft sixth edition of the WHO manual. The authors acquired new data from 1,789 males from five studies carried out in China, Egypt, Greece, Italy, and Iran. These new figures were combined with the reference value data used in the fifth edition, with a total of 3,589 specimens, providing new reference intervals in the upcoming $\mathrm{WHO}$ manual. Despite the number of specimens being twice as high compared to the previous WHO manual, the number of studies is still inadequate because certain geographical regions have not been represented. No study from South America has been included and only one research study has been carried out in the entire African continent. The number of participants is still insufficient, despite the significant increase compared to previous manuals, and the diversity in participants is also limited.

In conclusion, Esteves explained that the terminology in the upcoming sixth edition of the WHO manual used to describe low sperm concentration, motility, and morphology will also have to be qualified depending on the new reference values indicating conditions such as oligo-astheno-teratozoospermia. He suggested the clinicians who interpret SA should utilise the methods used within his institution and compare the patients' data with the lower (5\%), median (50\%), and upper (95\%) reference values provided by WHO manuals. A physical examination and clinical history should also be carried out to determine if additional tests, to further guide clinical management, and counselling are required. These thorough tests may reveal underlying conditions, which could be treated and potentially improve the health of patients and their offspring. 